\title{
Temperature Dependence of Ferroelectric Mode Frequency, Dielectric Constant and Loss Tangent in Deuterated TGSe Crystal
}

\author{
PRABHAT CHANDRA KHANDURI and TRILOK CHANDRA UPADHYAY* \\ Physics Department, H.N.B.Garhwal University (A Central University) \\ Srinagar-246174, Garhwal, Uttarakhant, India \\ prabhatkhanduri@yahoo.com
}

Received 17 November 2015 / Accepted 10 December 2015

\begin{abstract}
By fitting model values of physical quantities for deuterated TGSe crystal in theoretically derived expressions for ferroelectric mode frequency, dielectric constant and loss tangent in our earlier paper temperature dependences of these quantities have been calculated. Present results agree with experimental data of literature value.
\end{abstract}

Keywords: Ferroelectrics, Anharmonic, Dielectric constant, Loss tangent, Phase transition

\section{Introduction}

Triglycine selenate $\left(\mathrm{CH}_{2} \mathrm{NH}_{2} \mathrm{COOH}\right)_{3} \mathrm{H}_{2} \mathrm{SeO}_{4}$ crystal undergoes ferroelectric phase transition at $22{ }^{\circ} \mathrm{C}$. On deuteration the crystal does not show large shift on $\mathrm{T}_{\mathrm{c}}$. This shows that proton tunneling is not important in the phase transition mechanism in this crystal. The lattice parameters are $=9.54 \AA, b=12.92 \AA, c=5.86 \AA$ and $\beta=110^{\circ}$.

Experimental studies on TGSe and deutrated TGSe have been made extensively. Batra et al. ${ }^{1}$ have done pyroelectric study of TGSe and DTGSe crystals experimentally. Arago et al. ${ }^{2}$ have analyzed dielectric data for partially deuterated triglycine selenate crystals. Vorobyer $e t$ $a l^{3}$ have done relaxation processes studies of DTGSe crystals. Ghazaryan et al. ${ }^{4}$ have synthesized the DTGSe crystals. Choudhury and Chitra ${ }^{5}$ have done neutron diffraction study of TGSe crystal.

Theoretical studies on TGSe were initiated by Chaudhuri et $a l^{6}$. They considered twosub lattice pseudo spin model. They have used green's function method and obtained soft mode frequency, dielectric constant and $\mathrm{T}_{\mathrm{c}}$. Earlier researchers have not considered third order phonon anharmonic interaction. They have decoupled the correlations at an early stage. So that they could not produce better and convincing results. In our earlier paper, we have considered phonon anharmonic interactions terms. 
In the present study, we shall fit model values of physical quantities in the expressions obtained in our earlier paper $^{7}$ for deutrated TGSe crystal, Temperature dependence of ferroelectric mode frequency, dielectric constant and loss tangent will be calculated for DTGSe crystal. Theoretical results will be compared with experimental results of Hoshino et al. ${ }^{8}$ work.

\section{Calculation and Results}

In our earlier paper $^{7}$, two-sub-lattice pseudo spin-lattice coupled mode model along with third and fourth order phonon anharmonic interactions terms have been considered for TGStype crystals. The Green's function is differentiated twice with respect to time $t$ and $t$ ', with the help of modified Hamiltonian which is then set into Dyson's equation form. As a result Green's function was obtained as:

$$
G(\omega)=\pi^{-1} \Omega\left\langle S_{i}^{x}\right\rangle \delta_{i j}\left[\left(\omega^{2}-\hat{\Omega}^{2}\right)+2 i \Omega \Gamma(\omega)\right]^{-1}
$$

where

$$
\begin{aligned}
& \hat{\Omega}^{2}=\widetilde{\Omega}^{2}+\Delta(\omega), \\
& \widetilde{\Omega}^{2}=a^{2}+b^{2}-b c, \\
& a=2 J_{0}\left\langle S_{1}^{z}\right\rangle+K_{0}\left\langle S_{2}^{z}\right\rangle, \\
& b=2 \Omega \\
& c=2 J_{0}\left\langle S_{1}^{x}\right\rangle+K_{0}\left\langle S_{2}^{x}\right\rangle,
\end{aligned}
$$

$\Delta(\omega)$ and $\Gamma(\omega)$ are shift and width of response function (of Green's function). Values of $\Delta(\omega)$ and $\Gamma(\omega)$ have been given in our earlier paper ${ }^{7}$. By solving Eq. (2), the FE mode frequency was obtained as;

$$
\hat{\Omega}_{-}^{2}=\frac{1}{2}\left\{\left(\widetilde{\widetilde{\omega}}_{\mathrm{k}}^{2}+\tilde{\widetilde{\Omega}}^{2}\right) \pm\left[\left(\widetilde{\widetilde{\omega}}_{\mathrm{k}}^{2}-\tilde{\Omega}^{2}\right)^{2}+16 \mathrm{~V}_{\mathrm{ik}}\left\langle\mathrm{S}_{1}^{\mathrm{x}}\right\rangle \Omega\right]^{1 / 2}\right\},
$$

The dielectric constant $\varepsilon$ is related to susceptibility $\chi$ as $\varepsilon=4 \pi \chi$ (since in ferroelectrics $\varepsilon \gg 1$ ) which in turn is related to retarded Green's function given in Eq. (2) as $\chi=2 N \mu^{2} G(\omega)$. Therefore, putting the value of Green's function in Eq. (1), the value of dielectric constant $\mathcal{E}$ is obtained as:

$$
\in=\left(-8 \pi N \mu^{2}\right)\left\langle S^{x}\right\rangle\left(\omega^{2}-\hat{\Omega}^{2}\right)\left[\left(\omega^{2}-\hat{\Omega}^{2}\right)^{2}+4 \Omega^{2} \Gamma^{2}\right]^{-1},
$$

The dissipation of power when crystal is exposed to electromagnetic field is expressed as loss tangent. The loss tangent is the ratio of imaginary to real parts of dielectric constant:

$$
\tan \delta=\frac{2 \Omega \Gamma(\omega)}{\hat{\Omega}^{2}}
$$

By using model values of physical quantities for deuterated TGSe crystal, given in Table 1, temperature dependences of ferroelectric mode frequency, dielectric constant and loss tangent have been calculated and shown in Figures 1-3, 
Table 1. Model values of physical quantities for deuterated TGSe crystal

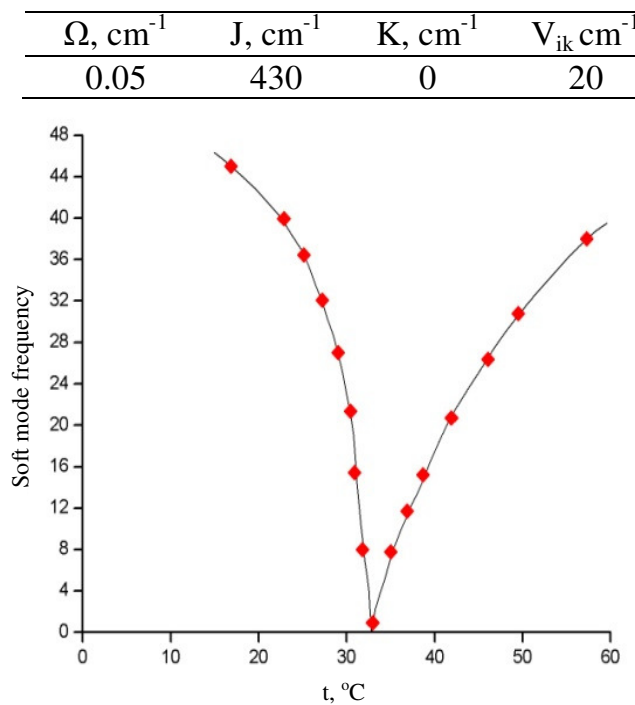

Figure 1. Calculated temperature ependence of soft mode frequency ( $\hat{\Omega}$ ) in deuterated TGSe crystal correlated with, $\operatorname{Exp}^{8}$

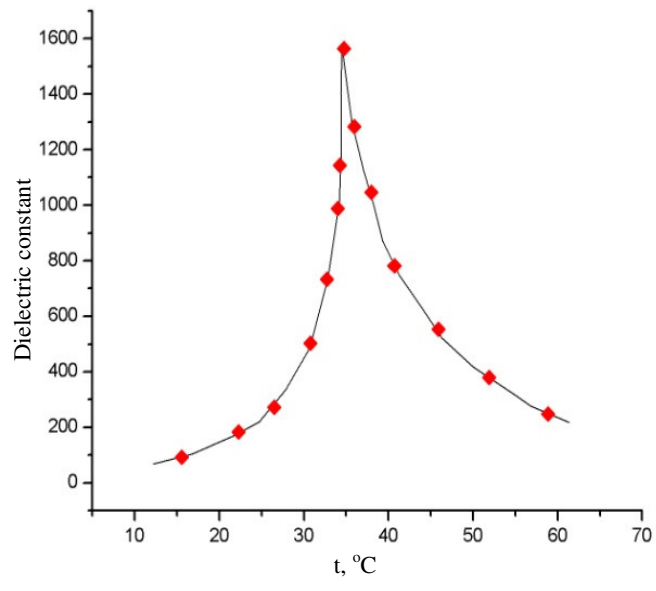

Figure 2. Calculated temperature dependence of dielectric constant $(\varepsilon)$ in deuterated TGSe crystal, $\operatorname{Exp}^{8} \nabla$

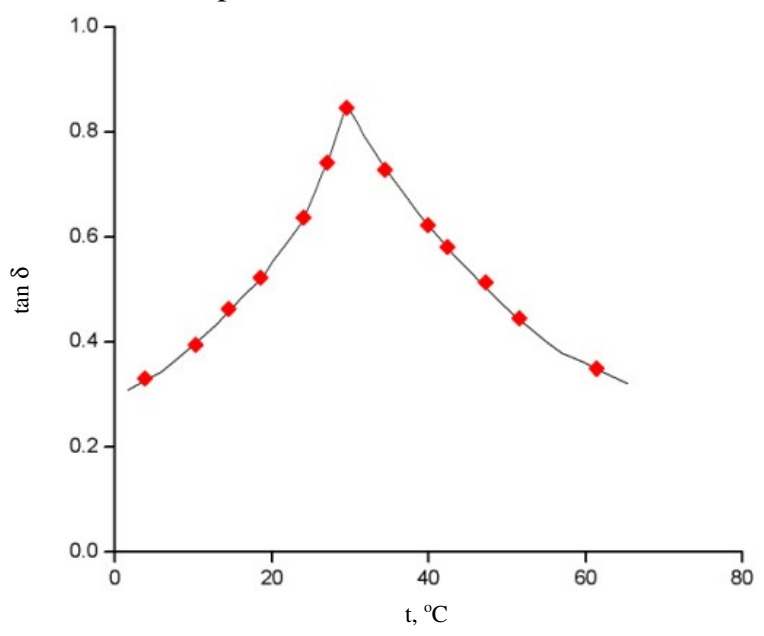

Figure 3. Calculated temperature dependence of tangent loss in deuterated TGSe crystal, $\operatorname{Exp}^{8}$

\section{Discussion}

In the present work, by fitting model values for deuterated TGSe crystal in the expressions obtained for crystal in our earlier paper ${ }^{7}$, the temperature dependence of ferroelectric mode frequency, dielectric constant and loss tangent has been calculated. Theoretically calculated results compare well with experimentally reported results of Hoshino et al. ${ }^{8}$ for deuterated TGSe crystal. 
The main aim of the present work is to explain isotope effect in DTGSe. On deuteration, the transition temperature shifts from $22{ }^{\circ} \mathrm{C}$ to $34{ }^{\circ} \mathrm{C}$ and both dielectric constant and loss tangent versus temperatures curves shift to quite new values. Our expression for transition temperature $T_{c}$ with values for DTGSe explains fairly isotope effect on $T_{c}$. Our expressions given in Eqs (8-9) explain temperature dependence of ferroelectric frequency, dielectric constant and loss tangent for DTGSe. The change in tunneling frequency is mainly responsible for isotope effects in DTGSe crystal. Hence, with the expressions obtained for DTGSe crystal and different values of physical quantities for DTGSe crystal, one can explain ferroelectric, dielectric and isotope effects in deuterated TGSe crystal.

\section{Conclusion}

Present study reveals that the modified model i.e. two sub lattice pseudo spin-lattice coupled mode model with addition of third and fourth order phonon anharmonic interactions terms explain quantitatively well the ferroelectric and dielectric behaviors of pure as well as deuterated TGSe crystal. Theoretical results agree with experimental results of Hoshino et al., ${ }^{8}$, which show the applicability of the present modified model for both TGSe and DTGSe crystals.

\section{Acknowledgements}

Authors are grateful to Eminent Physicist Prof B S Semwal (Former HOD) for his suggestions, Prof U C Naithani and Prof S C Bhatt (HOD), for their encouragements, Dr Sandeep Sharma and Dr Ashish Nautiyal for the discussions.

\section{References}

1. Batra A K, Bhattacharya S, Chilvery A K, Aggarwal M D, Edwards M E and Bhalla A S, J Photonics Energy, 2011, 1(1), 014001.

2. Arago C, Menendez L G and Gonzalo J A, Ferroelectrics, 2014, 462(1), 47-49; DOI:10.1080/00150193.2014.890853

3. Vorobyer A V, Gavrilova N D and Lotonov A M, Moscow University Physics Bulletin, 2014, 69(2), 175-179; DOI:10.3103/S0027134914020131

4. Ghazaryon V V, Fleck M and Petrosyan A M, Solid State Sci., 2012, 14(7), 952-963; DOI:10.1016/j.solidstatesciences.2012.04.036; Z Kristallographie, 2012, 227(9), 646665; DOI:10.1524/zkri.2012.1537.

5. Choudhury R R and Chitra R, J Phys Condes Matter., 2009, 21(33), 335901.

6. Chaudhuri B K, Choudhury K R and Banerjee Suvra, Phys Rev B, 1988, 38(1), 689; DOI:10.1103/PhysRevB.38.689

7. Upadhyay T C and Sharma Sandeep, Asian J Chem., 2011, 23(12), 5629-5631.

8. Hoshino S, Mitsui T, Jona F and Pepinsky R, Phys Rev., 1957, 107,1255. 